$R M x A C, \mathbf{5 3}, 180-189(2021)$

(c) 2021: Instituto de Astronomía, Universidad Nacional Autónoma de México

https://doi.org/10.22201/ia.14052059p.2021.53.37

\title{
A STRATOSPHERIC BALLOON FLIGHT PLATFORM AND ITS EMPLOYMENT IN GRAVITATIONAL WAVE COUNTERPART OBSERVATION EXPERIMENTS
}

\author{
László Mészáros ${ }^{1}$, András Pál ${ }^{1}$, Norbert Werner ${ }^{2,3}$, Masanori Ohno ${ }^{2,3,1}$, Gábor Galgóczi ${ }^{4}$, Jakub Ř́pa ${ }^{2}$, \\ Nagomi Uchida ${ }^{3,1}$, Naoyoshi Hirade ${ }^{3,1}$, Hiroto Matake ${ }^{3,1}$, László Kiss ${ }^{1}$, Jakub Kapuš ${ }^{5}$, Robert Laszlo ${ }^{6}$, \\ and Martin Koleda ${ }^{6}$ \\ RESUMEN
}

La iniciativa Cubesats Applied for MEasuring and LOcalising Transients (CAMELOT) propone desplegar un conjunto de nanosatélites de 3 unidades para localizar GRBs con cobertura total. La operación se basa en la medición del retardo. El retardo de tiempo del evento desencadenante entre satélites que de otro modo estarían distribuídos uniformemente alrededor de la Tierra en órbita terrestre baja (entre 500 y $600 \mathrm{~km}$ de altitud). En este diseño, los cristales de yoduro de cesio interactúan con radiación gamma emitiendo fotones ópticos. Mediante la utilización de este efecto, cada miembro de la flota está equipado con cuatro de estos centelleadores y los fotones emitidos son detectados por contadores de fotones de múltiples píxeles (MPPC). La sincronización precisa es crucial para este concepto, la marca de tiempo de los eventos y la sincronización por GPS. Para demostrar la viabilidad del concepto CAMELOT, un CubeSat de una sola unidad, llamado "GRBAlpha" se está desarrollando actualmente. GRBAlpha está equipado con un sólo bloque de centelleador pero los otros subsistemas son los mismos que estarán en las unidades CAMELOT. Describimos esta plataforma de una sola unidad-sistema, centrándonos en las versiones del modelo adecuadas para globos estratosféricos de gran altitud. Este modelo tiene un diseño estandarizado (que incluye configuración de pin-out, señalización y comunicación de bus) que es compatible con una proporción significativa de proveedores de sistemas CubeSat. Este sistema nuestro también es capaz de alojar múltiples cargas útiles al mismo tiempo, optimizando la utilización de experimentos con globos.

\section{ABSTRACT}

The Cubesats Applied for MEasuring and LOcalising Transients (CAMELOT) initiative proposes to deploy a fleet of $3 \mathrm{U}$ nanosatellites in order to localise GRBs with all-sky coverage. The operation is based on measuring the time delay of the event trigger between satellites that are otherwise uniformly distributed around the Earth in low-Earth orbit (between 500 - $600 \mathrm{~km}$ of altitude). In this design, caesium-iodide crystals interact with soft gamma radiation by emitting optical photons. Utilization of this effect, each member of the fleet is equipped with four of such scintillators and the emitted photons are detected by multi-pixel photon counters (MPPCs). Precise timing is crucial for this concept, the time-stamping of the events and the synchronisation is provided by GPS. In order to demonstrate the feasibility of the CAMELOT concept, a single-unit CubeSat, named "GRBAlpha" is currently being developed. GRBAlpha is equipped with a single block of scintillator but the other subsystems are all the same as it will be on the CAMELOT units. We describe this single-unit platform system, focusing on the model versions suitable for high-altitude stratospheric balloon flights. This model has a standardized layout (including pin-out configuration, signalling and bus communication) and compatible with significant proportion of CubeSat system vendors. This system of ours is also capable of hosting multiple payloads at the same time, optimizing the utilization of balloon experiments.

Key Words: balloons — instrumentation: detectors — X-rays: bursts

\footnotetext{
${ }^{1}$ Konkoly Observatory, Research Centre for Astronomy and Earth Sciences, Konkoly Thege Miklós út 15-17, Budapest H-1121, Hungary (meszaros.laszlo@csfk.mta.hu).

${ }^{2}$ MTA-Eötvös University Lendület Hot Universe Research Group, Pázmány P. st. 1/A, Budapest H-1117, Hungary.

${ }^{3}$ School of Science, Hiroshima University, Japan.

${ }^{4}$ Institute of Physics, Eötvös University, Pázmány P. st. 1/A, Budapest H-1117, Hungary.

${ }^{5}$ Spacemanic s.r.o., Slovakia.
}

\section{INTRODUCTION}

The discovery of the electromagnetic counterpart of gravitational waves has a significant impact in multi-messenger astronomy, hence the importance of detecting transient events is increasing. The difficulty in the detection is due to the fact that these

\footnotetext{
${ }^{6}$ NEEDRONIX s.r.o., Slovakia.
} 


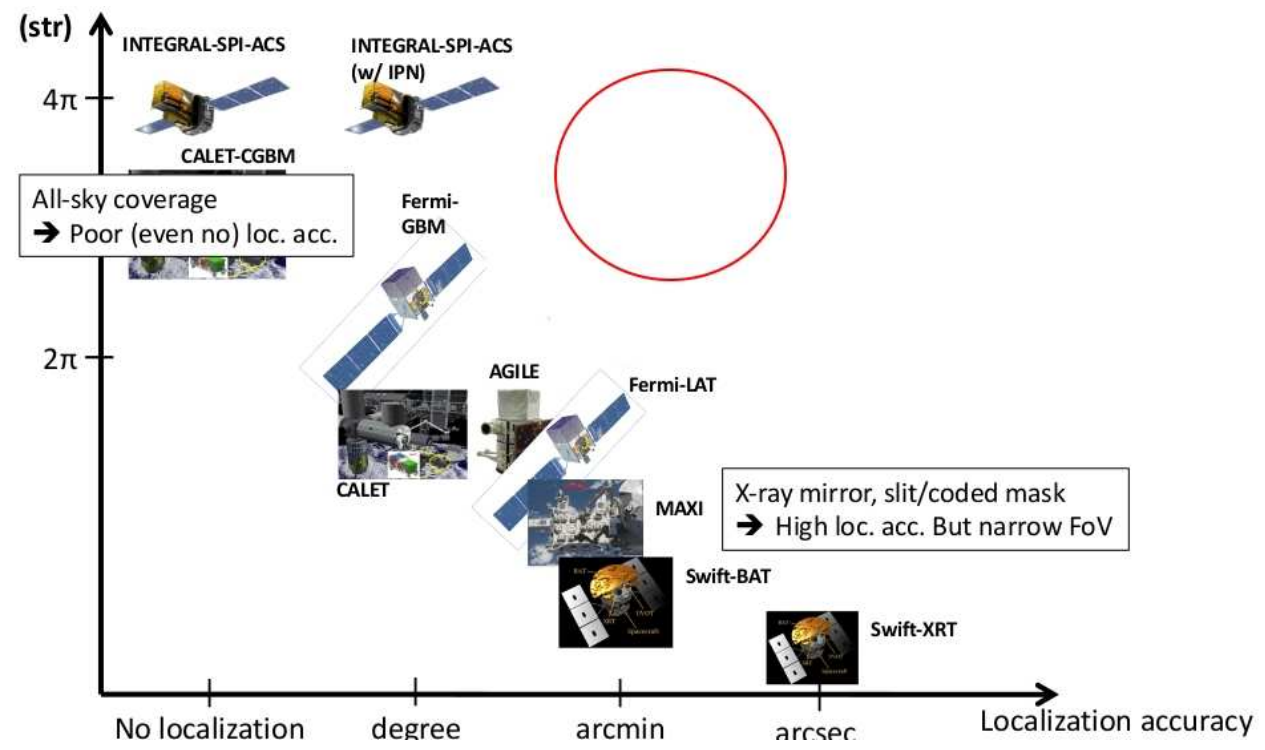

Fig. 1. A plot of the localisation accuracy of the most prominent gamma-ray detecting instruments with respect to their sky coverage. Currently the upper right corner (red circle) is empty which gap intended to be filled by CAMELOT with a localisation accuracy of $\sim 10^{\prime}$ all-sky coverage of at least 3 satellites.

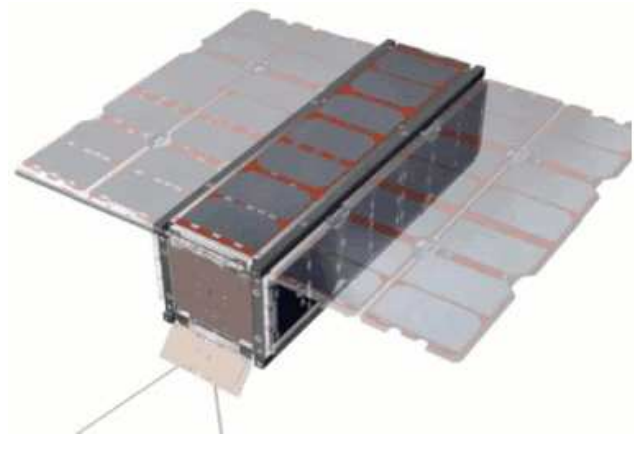

Fig. 2. An initial CAD image of the $3 \mathrm{U}$ version of CAMELOT.

events can be expected from any direction and at any moment. The solution can be the continuous monitoring with all-sky coverage. For instance, it has been proven (see Abbott et al. 2017; Goldstein et al. 2017) that the gravitational wave event GW170817 is originated from the merging of two neutron stars while this event was also visible for Fermi and independently a short gamma-ray burst GRB170817A was detected. To understand better the background physics of these extraordinary explosions not only detection is required but also good localisation in order to perform follow-up observations with rapid response telescopes on other wavelengths. Currently there are no instruments available that are capable of detecting and accurately localising gamma-ray bursts (see Figure 1) with high sky coverage at once. This gap is intended to be filled by the Cubesats
Applied for MEasuring and LOcalising Transients (CAMELOT) mission. (Ohno et al. 2018; Pál et al. 2018; Řípa et al. 2018; Torigoe et al. 2019; Werner et al. 2018).

The CAMELOT design is going to be capable of detecting not only short and long gamma-ray bursts, but also other phenomena that are producing gamma-ray emission such as core collapse of massive stars or magnetar outbursts. In addition, it is also feasible to detect terrestrial gamma-ray flashes which are sourced by thunderstorms in the Earth's atmosphere. In order to ensure of the mission success and validate the detector design at the same time, first we are developing a $1 \mathrm{U}$ sized version of the satellite system - named GRBAlpha - for technical demonstration. Prior to space deploy we plan to run a series of high-altitude balloon experiments which can be a good intermediate step for testing either the subsystem or the integrated unit. In this paper, first, we briefly present the actual status of the CAMELOT initiative (Sec. 2) and the main concepts of the GRBAlpha experiment (Sec. 3). Following these, the balloon experiment design is described in Sec. 4, focusing not just on GRBAlpha itself but the possibilities of integrating another types of experiments by employing the same platform. Our results are summarized in Sec. 5 .

\section{CAMELOT}

CAMELOT is proposed to be a constellation of $3 \mathrm{U}$ nano-satellites (see Figure 2), distributed uni- 


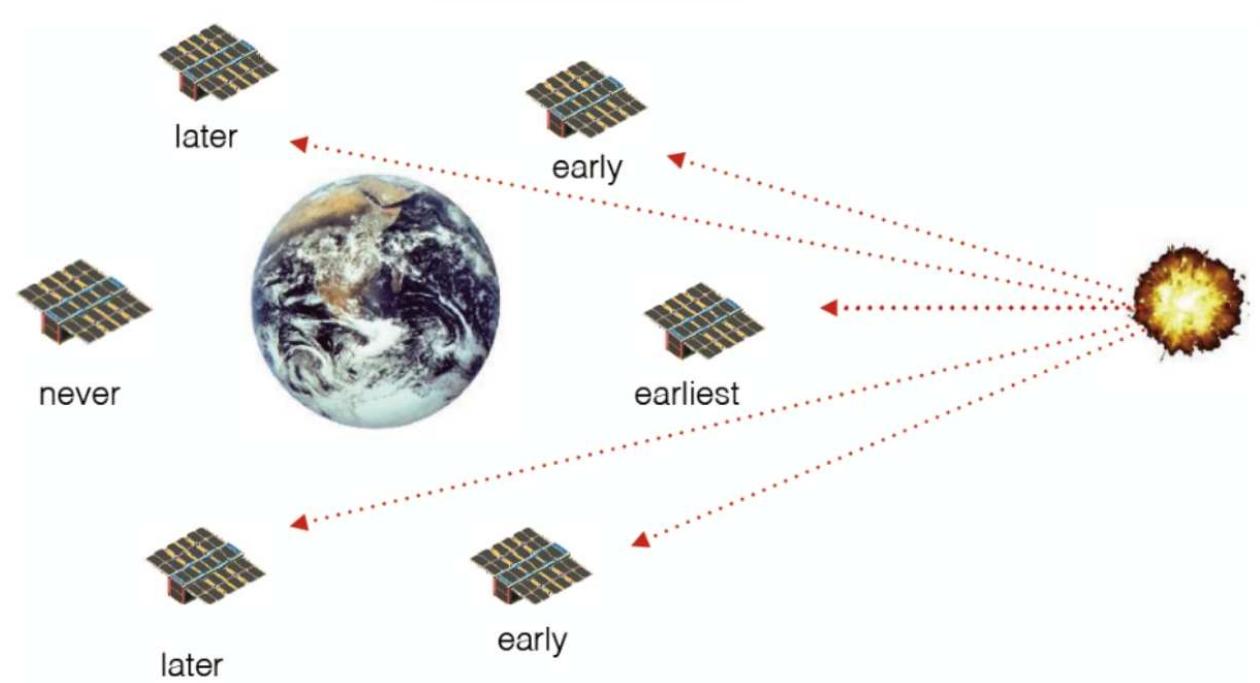

Fig. 3. The concept of operation of Camelot. By accurately measuring and time-stamping the signal arrival time we can determine direction of the source with triangulation.

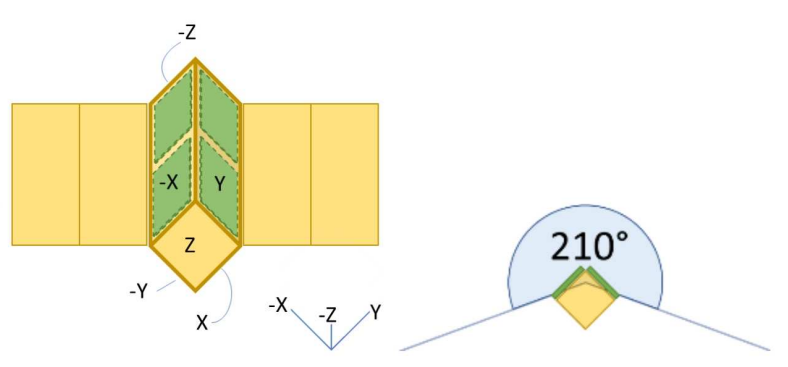

Fig. 4. By mounting the detectors on two perpendicular side of the satellite the field-of-view can be increased to $210^{\circ}$ for each unit.

formly on low-Earth orbit. The mission concept is based on the measurements of the time delay of the event trigger between the individual units, as it is displayed in Figure 3.

With such a triangulation-alike method, the direction of the source can be determined. Each of the CubeSats is equipped with four separate, $150 \times 75 \times 5 \mathrm{~mm}$ caesium-iodide (CsI) scintillator crystals (Figure 4, green areas) which are emitting optical photons whenever they have been hit by soft gamma radiation. The short side of the scintillator, two independent channels of four multi-pixel photon counters (MPPCs) connected in parallel are mounted to capture the optical photons. These MPPCs, along with another auxiliary electronics and connectors are supported by a small, $75 \times 5 \mathrm{~mm}$ PCB in accordance with the cross-section of the scintillator.

\subsection{GPS module}

Timing based localisation requires high timing accuracy for that purpose we utilize GPS satellites (see Figure 6). As it has been proven (Pál et al. 2018), the system is going to be capable of performing time-stamping for event triggers with a timing accuracy and precision better than 0.02 millisecond (Figure 7). This configuration yields an all-sky coverage with $\sim 10^{\prime}$ localisation accuracy (Ohno et al. 2018).

\subsection{Background radiation}

The CAMELOT units are intended to orbit in $\sim 500 \mathrm{~km}$ height where various type of high energy particles are present. We used AP-9 and AE-9 models (Ginet et al. 2013) developed by ESA to reveal the environment in which CAMELOT operates. However, these models are imperfect since they do not contain numerous other particles. For this purpose we used the measurements of other space missions and we performed Geant4 simulations with the aforementioned spectrum to estimate the background radiation that can be expected in these orbits. Another important issue is the high flux of radiation when the satellites pass through the South Atlantic Anomaly and the polar regions (in the case of $i=97.2^{\circ}$ orbit, see Figure 8), which can cause the degradation of the MPPCs. In order to reduce the ionizing dose, a lead shielding must be added to the aluminum support structure. Hence, the casing provides not only mechanical protection but effectively reduces the degradation effects of both trapped protons and electrons. 

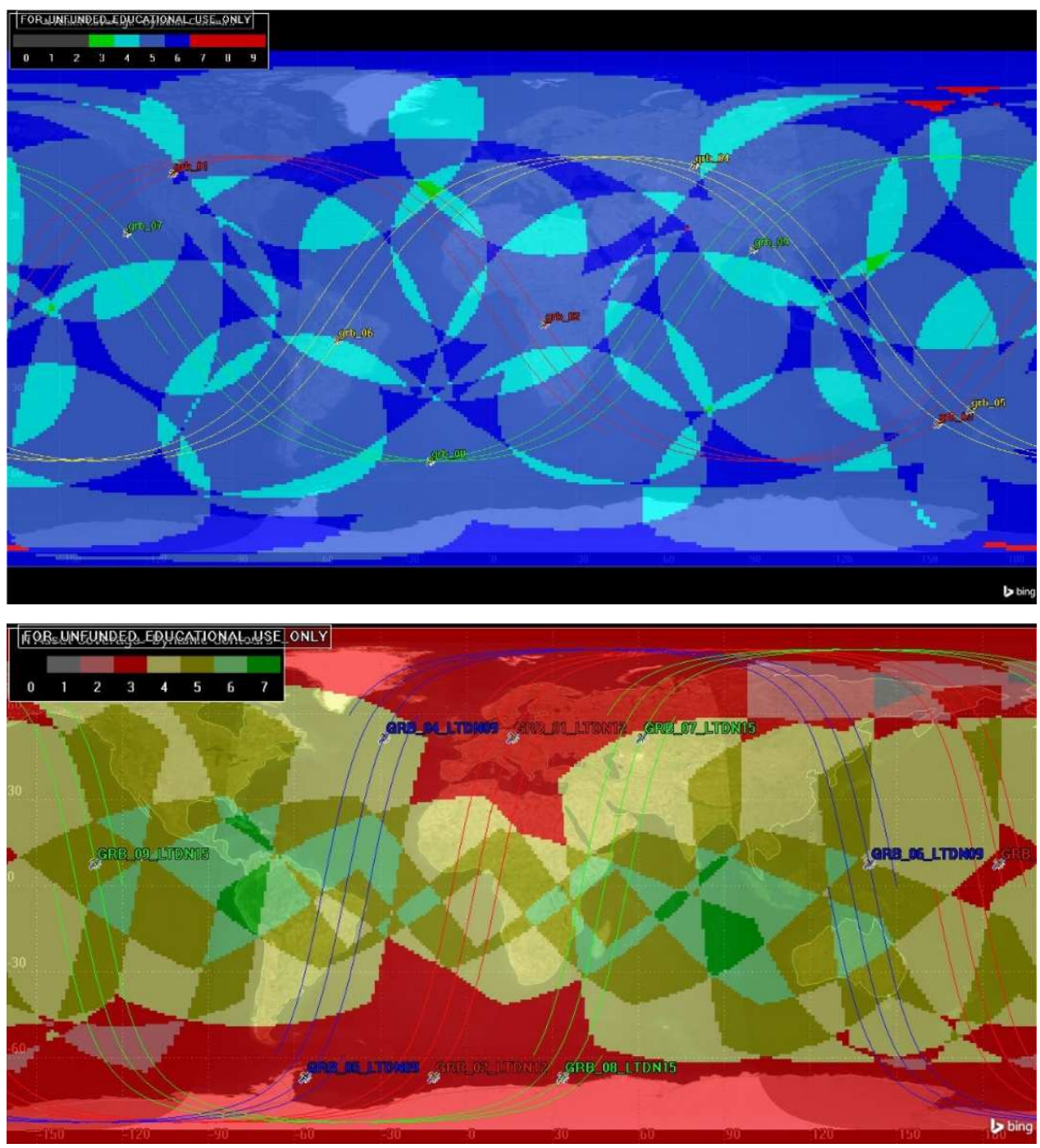

Fig. 5. Expected sky coverage for the two possible orbit: sun-synchronous polar orbit and $i=53^{\circ}$ orbit. In both cases at least 3 of the whole constellation would cover a particular area.

A detailed description of this study is given by Řípa et al. (2019).

\section{GRBALPHA}

In order to demonstrate the feasibility of the mission concept first we develop a $1 \mathrm{U}$ prototype version of CAMELOT, named GRBAlpha (see Figure 9). In the case of GRBAlpha we use 1/8 detector area of the CAMELOT goals, namely a single block of $75 \times 75 \times 5 \mathrm{~mm}$ sized scintillator. Otherwise, all of the other subsystems - including analog and digital MPPC readout electronics, data acquisition, triggering and time-stamping - were designed to be capable of operating in both versions.

For GRBAlpha we intended to use off-the-shelf frame structure which implies various mechanical constraints on the support structure of the detector and therefore on the whole setup. Due to the limitation on the lateral extension of the CubeSat standards and the form factor of such frame structures, the only possibility is to mount the detector on the top side of the satellite, $(Z+$, see CubeSat Design Specification document ${ }^{7}$, see also Figure 10). Since the trio of the scintillator-casing-shielding has a relatively large mass this causes a large displacement on the centre of mass in the $Z$ direction but it has minimal effect in the $X$ and $Y$ directions thus the balancing is manageable by placing the batteries on the opposite side.

The payload electronics controls the readout of the MPPCs. First, the operation of the sensors re-

\footnotetext{
${ }^{7}$ https://www.cubesat.org/resources
} 


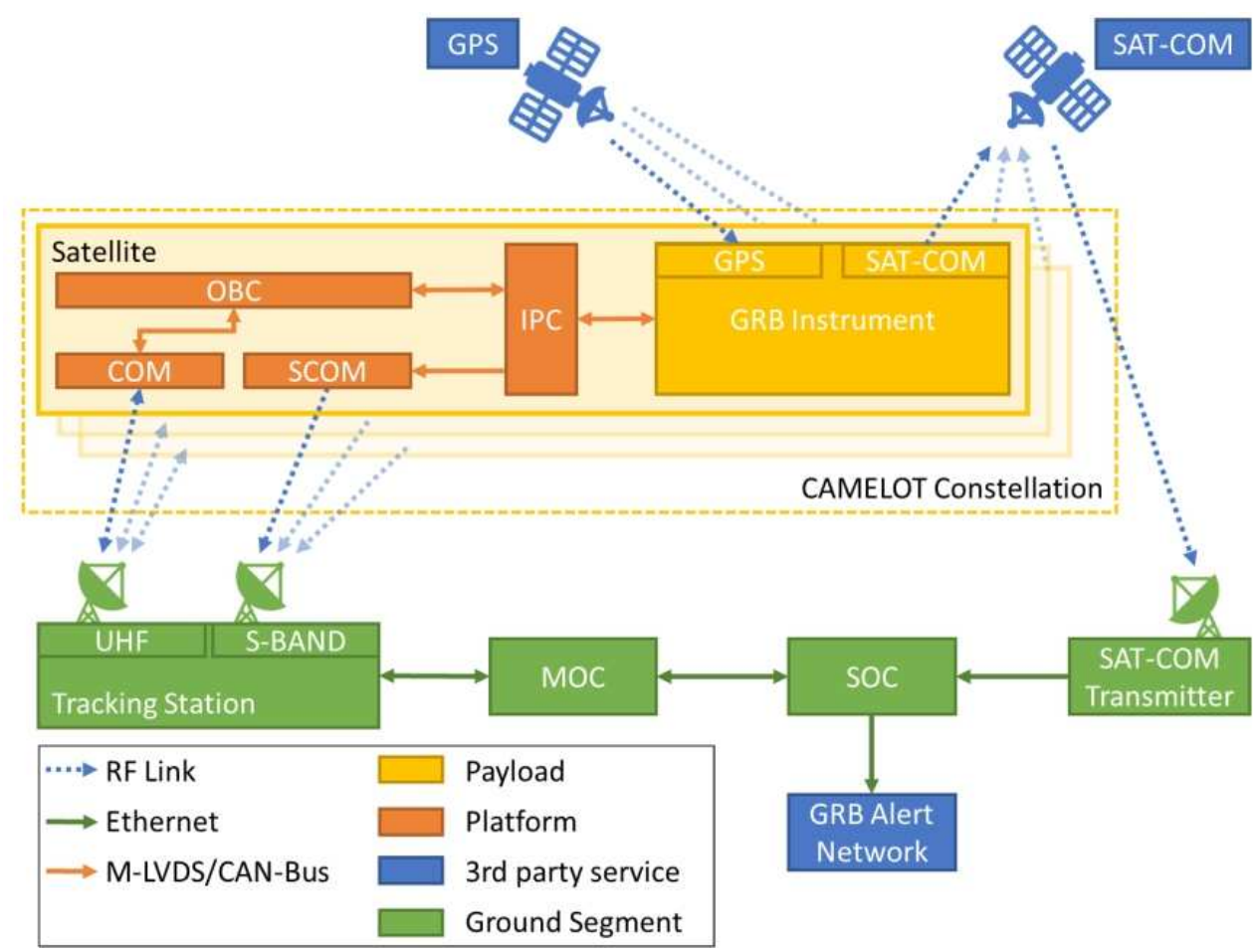

Fig. 6. Block diagram of the CAMELOT mission. Each satellite communicates and transfer data with S-band and UHF and/or via SAT-COM.

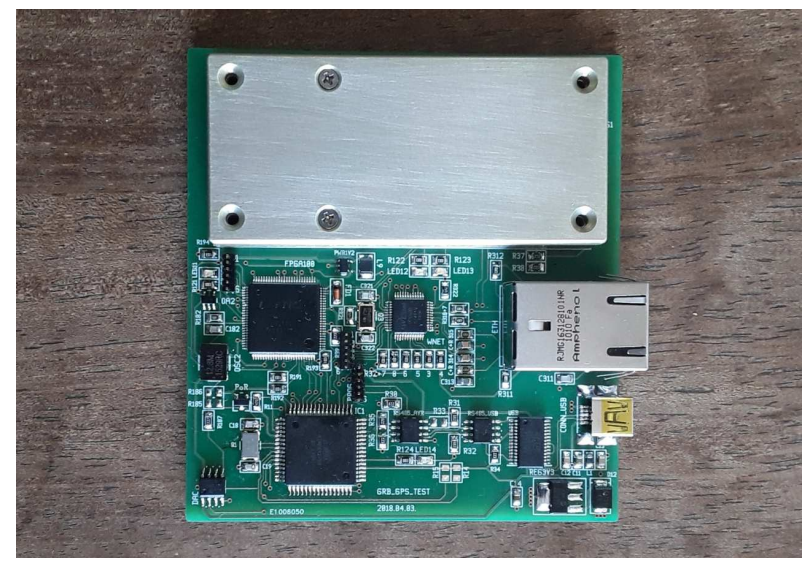

Fig. 7. A prototype board of the GPS receiver and timestamping unit as implemented in a form-factor similar to the CubeSat standards. Precision and accuracy of this implementation can be verified by several independent manner such as additional GPS receivers and Network Time Protocol.

quires $\sim 55 \mathrm{~V}$ which is regulated by a boost DC/DC power converter. The analog output of the sensor is filtered with RC filters before the charge shaping amplifier. This is followed by CR-RC pulse shaping of the signal which then passed to a 12-bit, 5MSPS serial sampling analog-to-digital converter. The de- scribed electronics are implemented in one daughterboard PCB (see Figure 11). The payload motherboard host two of such individual readout and ADC daughterboards for the separate channels. The digital data processing is done by a single FPGA with capacity of 7680 logic cells and 32x4096bit embedded block RAMs which is sufficient to implement the date processing of the dual channel acquisition.

The telemetry, on-board computer (OBC) and the attitude determination system (ADS) modules for GRBAlpha are based on the flight heritage of the SKCube, developed by the Slovak Organisation for Space Activities $\left(\mathrm{SOSA}^{8}\right)$. The GPS module is ready and tested (see Section 2.1.) with a designated prototype board, only circuit simplification and layout modification is required in order to be compatible with the other subsystems and $\mathrm{PC} / 104$ standard.

It is important to note that the $\mathrm{PC} / 104$ standard defines the form factor of a 104 pin header that connects the individual subsystems. During the development we checked the available OBCs of various vendors (GOMspace, ISIS, ENDUROSAT, CUBEspace, etc.) and we standardized the header pin-out configuration in order to be compatible with these systems.

\footnotetext{
${ }^{8}$ http://sosa.sk
} 

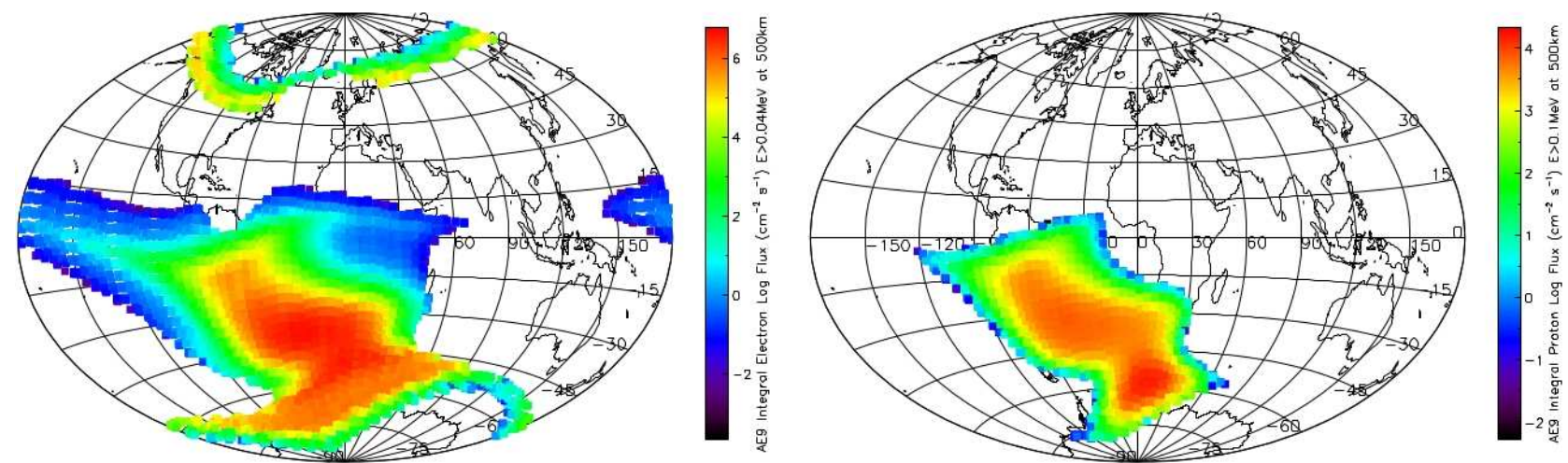

Fig. 8. Integral flux of the geomagnetically trapped electrons (left) and protons (right) according to the AE9 and AP9 models. Outside of the South Atlantic Anomaly the effect of high energy protons is negligible while the flux of high energy electrons can be relevant in the polar regions. Outside of these regions the space environment can be considered as ideal for detecting gamma-ray transient events.

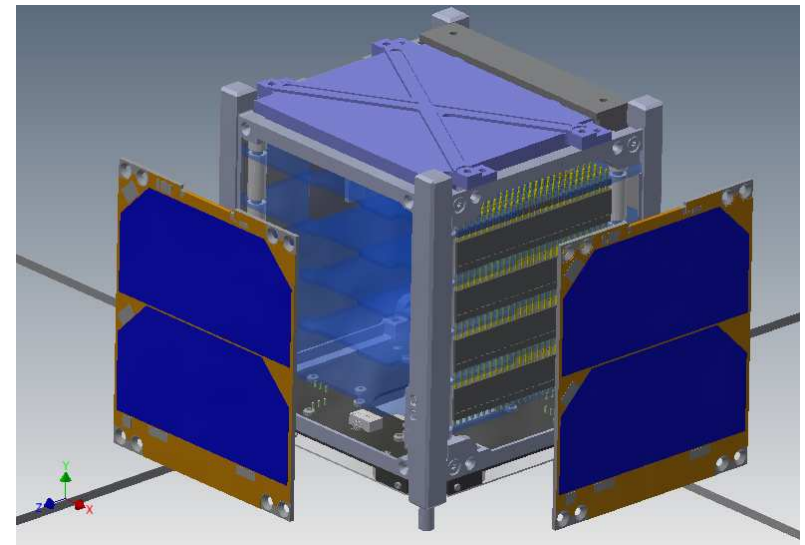

Fig. 9. A nearly complete CAD model of GRBAlpha. On the top side the scintillator casing is located where originally a solar panel is prepared to be mounted. By replacing the solar panel with the detector means a loss in the power budget. We considered to place the scintillator below the solar panel but its stopping power results a $30 \%$ loss in the measured signal.

\section{HIGH ALTITUDE BALLOON EXPERIMENTS}

Before the era of space telescopes, gamma-ray detection was only feasible by mounting the detector on high altitude balloons and release them to the stratosphere where atmospheric absorption is negligible (Haymes et al. 1968). For an initial technical demonstration, one can mount GRBAlpha on high-altitude meteorological balloons. Such balloons are capable of reaching an altitude of $35-40 \mathrm{~km}$ where the ambient conditions are suitable for testing the operation of the integrated system - including GPS, telemetry and payload modules. For the balloon-borne experiments we will use relatively small weather balloons with a payload mass of $\sim 2-3 \mathrm{~kg}$. This setup will
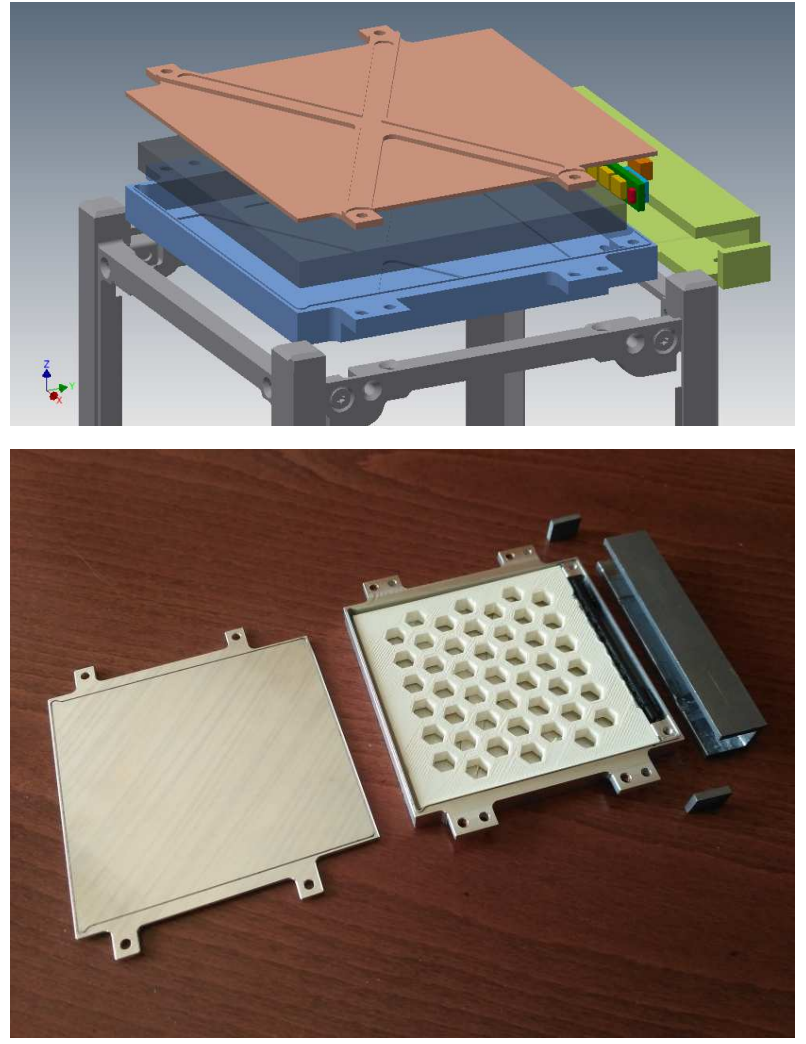

Fig. 10. CAD (upper) and manufactured (lower) pictures of the designed aluminum support structure

be capable of flying up to $\sim 7$ hours. Assuming a single GRB per day, such flight duration yields a detection probability around $10-15 \%$. In the current status, performing such trials is easy to arrange and cost-effective compared to satellite deployment and 


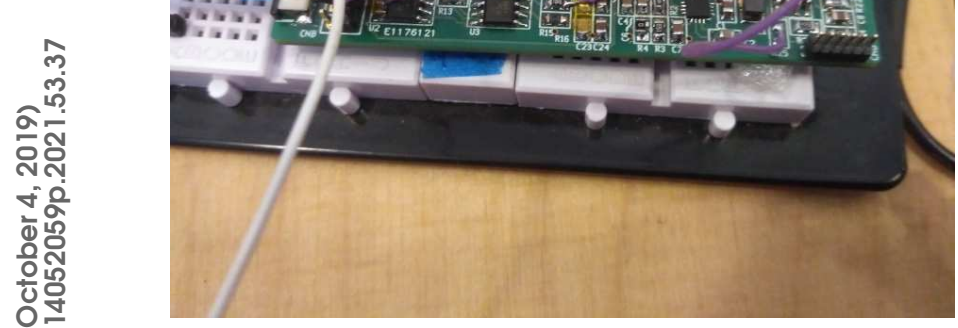

Fig. 11. The readout electronics for an individual channel in the laboratory during testing and integration.

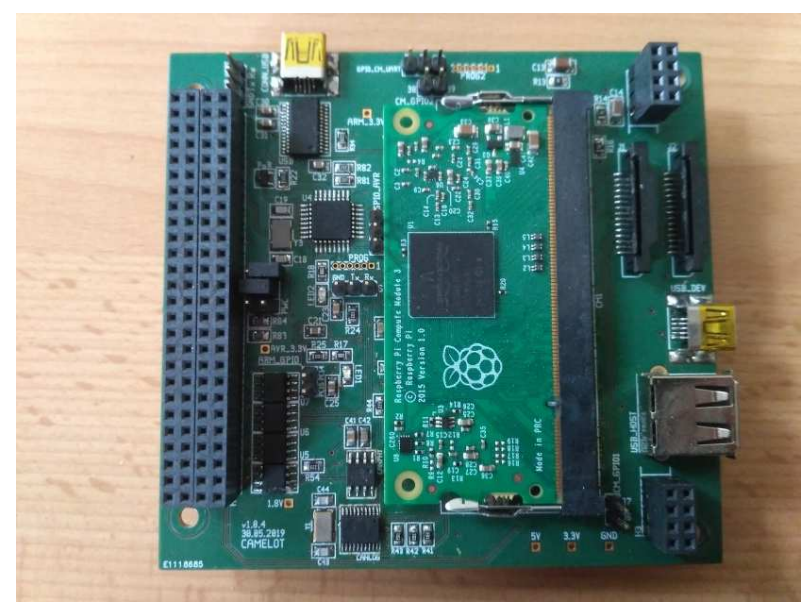

Fig. 12. The PC/104 form factor on-board computer with the Raspberry PI Compute Module in its core. For the balloon-borne experiment this setup simplifies the data acquisition since the module is capable of running a native Linux. We added an ARM core and a microcontroller to supervise, perform housekeeping and redundant resetting purposes.

it can reveal hidden mistakes in the concept or in the design.

\subsection{On-board computer module}

For the balloon-borne experiment we designed an individual on-board computer of which the core is a Raspberry PI Compute Module (see Figure 12). The module is capable of running native Linux onto which we can just simply save all data using SD cards as storage. The module is also has two camera interfaces to which we attach standard or wide-field external camera modules.

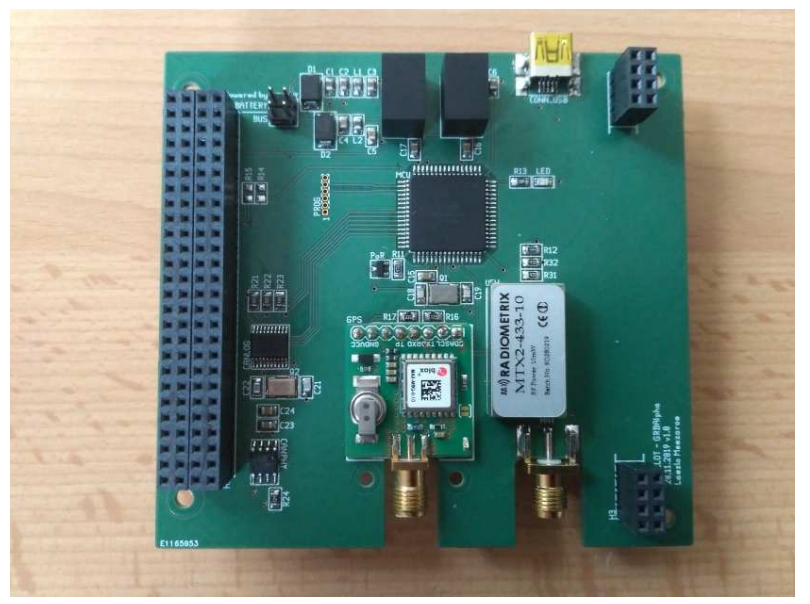

Fig. 13. The utilization of "Pi in the Sky" components simplifies the design and the usage of the telemetry board for the high altitude balloon experiments. We mounted the GPS module as a daughterboard and use it with with an active receiver antenna via an SMA connector.

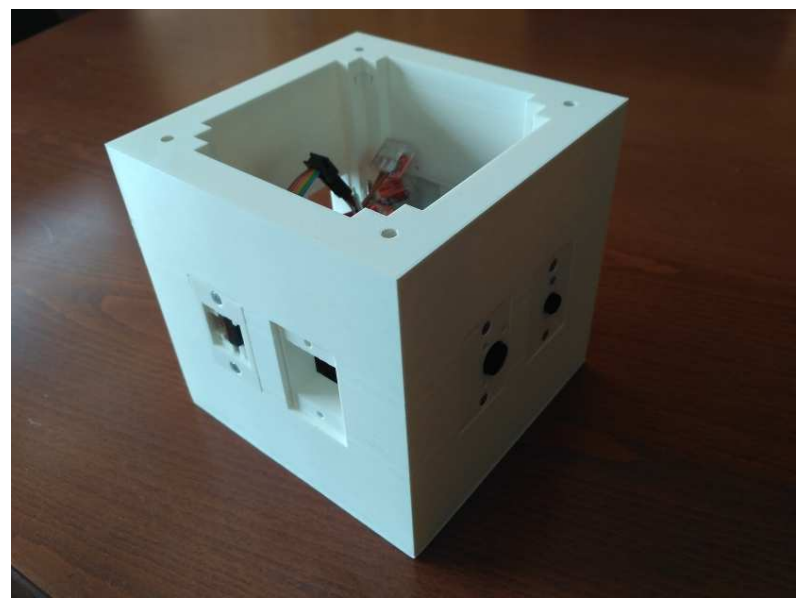

Fig. 14. 3D printed high altitude balloon gondola for a $1 \mathrm{U}$ CubeSat. Into the windows various type of peripherals can be installed. The gondola also provides thermal isolation due to the infill pattern.

\subsection{Telemetry module}

In order to simplify the design we utilize the "Pi in the Sky" uBLOX breakout board for the GPS receiver and a radio transmitter module (see Figure 13) which are designed for tracking high altitude balloons. The data acquisition and transmitter control is done by an ATmega128 microcontroller, having two independent serial interfaces required by the GPS and transmitter modules.

\subsection{Carrier gondola}

The payload carrier is a 3D printed gondola (Figure 14) into which a completely assembled CubeSat 


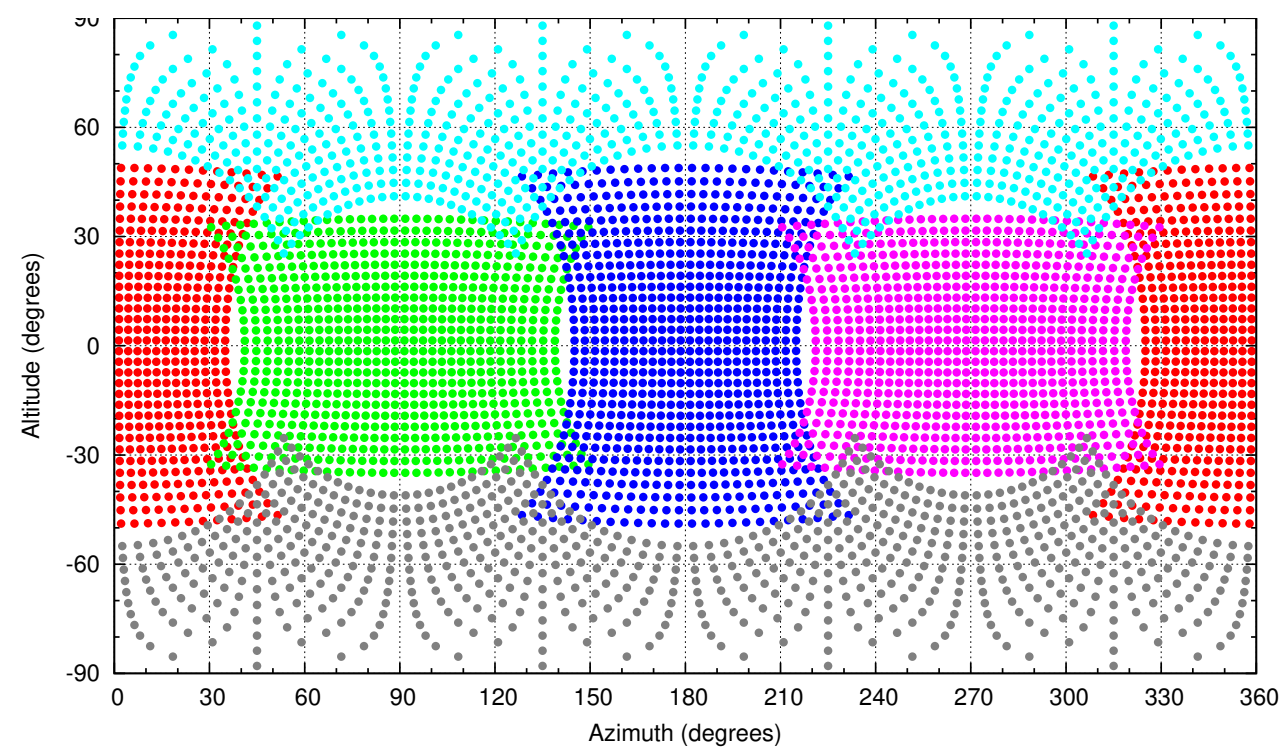

Fig. 15. Full-sky projection of six perpendicular MLX90640 sensors and the corresponding pixel centroid coordinates in an altitude-azimuth coordinate system.
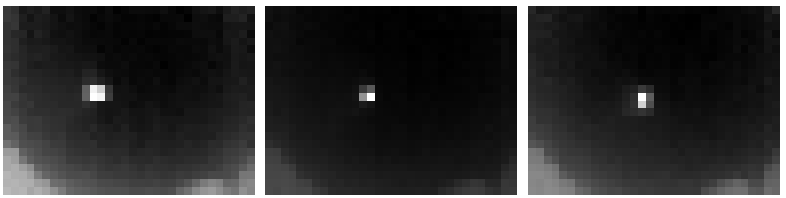

Fig. 16. Some example thermal images of the sky, taken by a fixed MLX90640 sensor as the Sun moves in front of it. The frames are separated by $\sim 17$ minutes in time. While the horizon is not covered fully in this setup, it can clearly be seen at the bottom of these images.

or simply electronics supported by PC/104 compatible mounting holes can be installed. On each side of this cube two $30 \mathrm{~mm} \times 30 \mathrm{~mm}$ windows were added where a variety of peripherals can be inserted. The aforementioned RPi camera modules are mounted in the windows of two opposite sides while the GPS antenna is located on the top side and a "remove before flight" switch on the bottom side. We also propose an experiment of determining the attitude of the gondola by mounting an IR sensor on each side (see also Sec. 4.4). Besides the mechanical protection, this gondola works as thermal isolator since the so called "infill" of the printing is built up from a pattern of summit set cubes.

\subsection{Attitude determination}

While in the case of a $3 \mathrm{U}$ CubeSat, accurate attitude control is feasible by involving currently available technologies, $1 \mathrm{U}$ CubeSats have less possibilities for attaining attitude control. Mainly, such CubeSats are stabilized either by actively (magne-

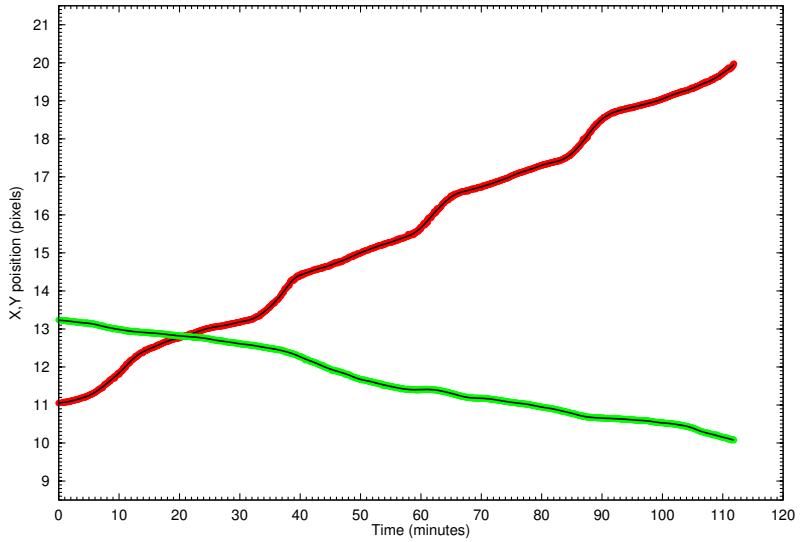

Fig. 17. Absolute pixel coordinates of the best-fitted centroid position corresponding to the Sun as seen by an MLX90640 sensors during a run of roughly 110 minutes. In this ground-based experiment, the sensor was fixed while the apparent motion of the Sun reflected the rotation of Earth. The red curve corresponds to the $x$ pixel coordinates while the green one is for the $y$ pixel coordinates. The best-fitted spline curves are superimposed as black. The residuals are in the order of few hundreds of a pixel.

torquers) or passively (by permanent magnets), depending on the power budget and/or the available space within the CubeSat frame. In the case of the GRBAlpha experiment, an active attitude control is not a requirement, however, it is highly valuable to have a knowledge about the attitude with an accuracy of a few degrees. This is important because of the properly interpretation of the MPPC signals 


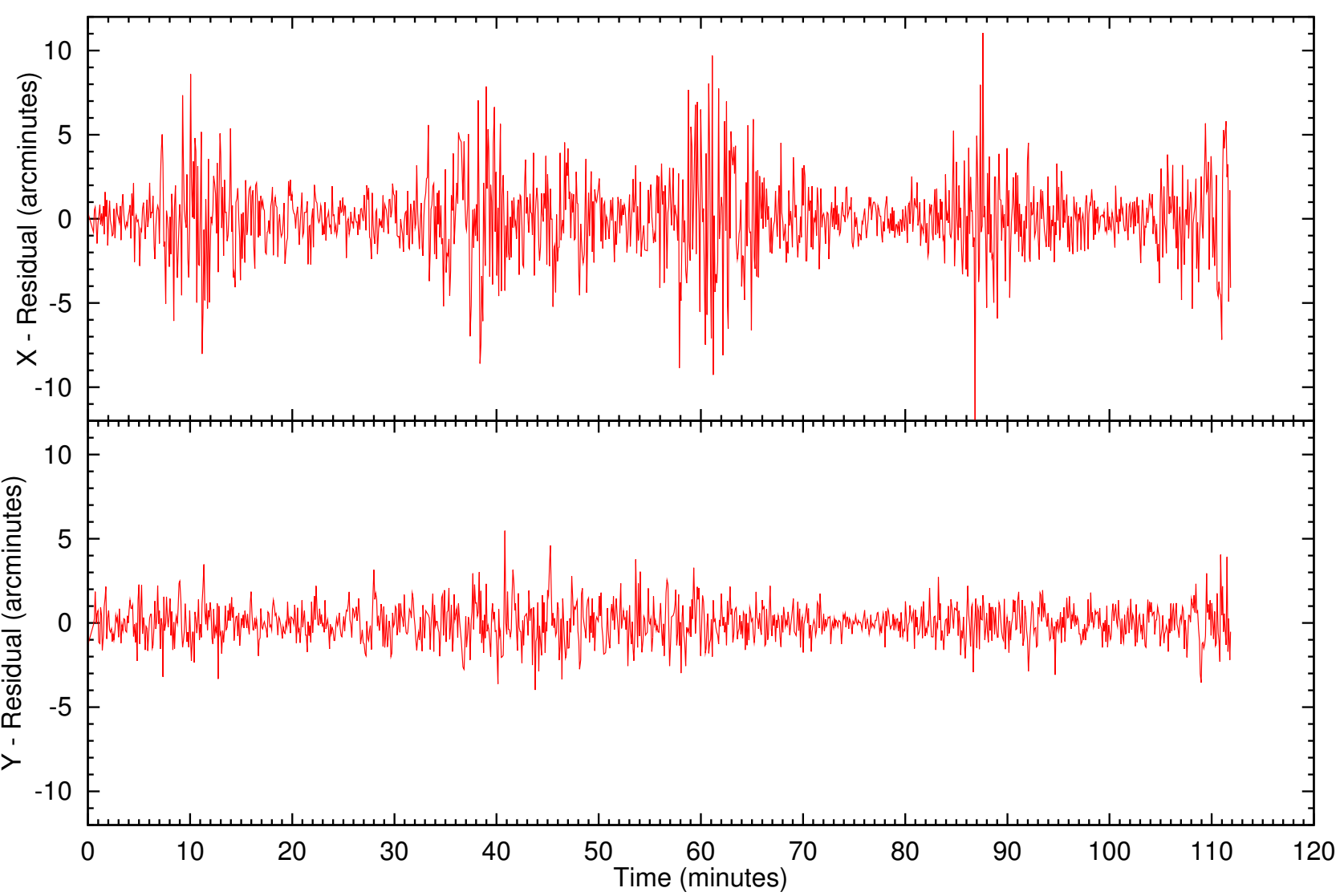

Fig. 18. The $x$ and $y$ residuals of the spline fitting procedure corresponding to the experiment. The typical residuals in the order of a few hundreds of a pixel which is equivalent to a few seconds of arc on the sky.

via the computation of the effective area of the CsI detectors.

In order to recover the attitude, we investigated the usability of MLX90640 ${ }^{9}$ type high-resolution thermal imaging sensors for such a purpose. The operation principle is simple: we measure the infrared radiation of the Sun and the apparent horizon using thermal images with an effective resolution of $32 \times 24$ pixels. For these particular sensors, the image refresh rate is dynamically adjustable from 0.5 to $64 \mathrm{~Hz}$ (which is otherwise equivalent to $0.25-32$ frames per second since the readout is interleaved). By considering the $110^{\circ} \times 75^{\circ}$ field-of-view of the sensor it can be shown that with proper arrangement of six sensors it is possible to provide a (nearly) full-sky coverage (see Figure 15). This is feasible by mounting a sensor on each side of a cube, for instance a CubeSat or the gondola of the high balloon experiment. The control and data acquisition of these sensors are manageable via $I^{2} C$ interface. While it is possible to connect all of the sensors on the same

\footnotetext{
${ }^{9}$ https://www.melexis.com/en/product/mlx90640/farinfrared-thermal-sensor-array
}

bus, however we should consider to separate into two or more channels and duplicate the supervision over the bus in order to ensure redundant operation.

In order to characterize the precision of these sensors we set up an experiment by fixing a sensor to the ground and continuously observing the position and the apparent motion of the Sun. By obtaining a series of images (see Figure 16) with a cadence of $5 \mathrm{sec}$ for two hours and extracting the centroid pixel coordinates (see Figure 17) we are able to determine the precision by fitting a spline function on the data and calculated the residuals (Figure 18). The residuals in the range of hundredths of a pixel, which is equivalent to a few seconds of arc on the sky.

\section{SUMMARY}

The Cubesats Applied for MEasuring and LOcalising Transients initiative is a mission for detecting and localizing of gamma-ray bursts - which are proven to be the electromagnetic counterparts of gravitational waves - and other transient highenergy astrophysical events. In order to successfully set up the full constellation we intend to demonstrate 
the concept in a step-by-step fashion. First, we use a set of high-altitude weather balloons to reach the stratosphere and test the individual subsystems. Even with such balloon-borne experiments we have a detection probability of $\sim 10-15 \%$. The next step is to deploy a single unit version - named GRBAlpha - of CAMELOT into low-Earth orbit and demonstrate operation feasibility. This is followed by a $3 \mathrm{U}$ final version and the full constellation in the next few years. During the development of GRBAlpha we started a spin-off project for determining the attitude of a $1 \mathrm{U}$ CubeSats as an alternative method. We intended to use an arrangement of six infrared sensors on the side of a cube and observing the position of the Sun and the horizon as a reference from which the attitude can be derived. While initial studies have shown that the precision and accuracy of such an attitude determination system is sufficient for the GRBAlpha needs, this concept still needs to be verified in a balloon flight.

Acknowledgements: This project is supported in part by the GINOP-2.3.2-15-2016-00033 grant which is funded by the Hungarian National Research,
Development and Innovation Fund together with the European Union. Additional funding is received via the grant KEP-7/2018 of the Hungarian Academy of Sciences.

\section{REFERENCES}

Abbott, B. P., Abbott, R., Abbott, T. D. et al. 2017, PhRvL, 119, 1101

Ginet, G. P., O'Brien, T. P., Huston, S. L. et al. 2013, SSRv, 179, 579

Goldstein, A., Veres, P., Burns, E. et al. 2017, ApJ, 848, 14

Haymes, R. C., Ellis, D. V., Fishman, G. J., Kurfess, J. D., \& Tucker, W. H. 1968, ApJ, 151, 9

Ohno, M., Werner, N., Pál, A., et al. 2018, SPIE, 10699, 64

Pál, A., Mészáros, L., Tarcai, N., et al. 2018, arXiv:1806.03685

Řípa, J., Werner, N., Pál, A., et al. 2018, 69th International Astronautical Congress, IAC-18, B4, 2, 8, x46335

Řípa, J., Galgóczi, G., Werner, N., et al. 2019, AN, 340, 666

Torigoe, K., Fukazawa, Y., Galgóczi, G., et al. 2019, NIMPA, 924, 316

Werner, N., Řípa, J., Pál, A., et al. 2018, SPIE, 10699, 2 